\title{
EXPERIÊNCIA EM GRUPOS DE CONVIVÊNCIA DE IDOSOS: INTERFACES COM A TERAPIA OCUPACIONAL
}

\section{Experience of social groups for older people: interfacing with occupational therapy \\ Experiencia de grupos de convivencia con mayores: interfaces de la terapia ocupacional}

\author{
Marciane Montagner Missio iD \\ Universidade Franciscana - UFN - Santa Maria (RS) - Brasil \\ Silvani Vargas Vieira iD \\ Universidade Federal de Santa Maria - UFSM - Santa Maria (RS) - Brasil
}

\section{RESUMO}

Objetivo: Descrever a experiência de acadêmicas de Terapia Ocupacional na inserção de um grupo de convivência de idosos em uma comunidade localizada na região central do Rio Grande do Sul, Brasil. Síntese dos dados: A ação foi desenvolvida no Estágio Supervisionado em Saúde da Comunidade, no período de março a julho do ano de 2015, realizado no sexto semestre do curso de Terapia Ocupacional. Na atuação dos acadêmicos com os participantes do grupo de convivência, foram realizadas atividades visando à integração de ações de ensino, pesquisa e extensão universitária, com foco na melhoria da qualidade de vida e condição de saúde das participantes através da prevenção de agravos e complicações, considerando as doenças crônicas não transmissíveis (DCNT) apresentadas pela maioria dos participantes do grupo de idosos. Conclusão: As experiências evidenciaram que os grupos de Terapia Ocupacional permitiram a identificação de potencialidades e habilidades de uma população pouco valorizada produtivamente na sociedade, de forma que o grupo se constituiu como um espaço de ressignificação de vidas, superação de dificuldades cotidianas, aquisição de hábitos de vida saudáveis e ajuda para um envelhecimento ativo.

Descritores: Terapia Ocupacional; Idoso; Prática de Grupo; Educação em Saúde.

\begin{abstract}
Objective: To describe Occupational Therapy students' experience of their participation in a social group for older people in a community located in the central region of Rio Grande do Sul, Brazil. Data synthesis: The activity was developed during the Supervised Internship in Community Health from March to July 2015 in the sixth term of the Occupational Therapy course. Students' work with the participants of the social group included activities aimed at integrating university teaching, research and extension activities focused on improving the quality of life and the health status of the participants through the prevention of injuries and complications taking into consideration the noncommunicable diseases (NCDs) presented by most of the participants in the group. Conclusion: The experience showed that Occupational Therapy groups enabled the identification of potential and skills in a population that is undervalued by society in terms of productivity and that the group constituted a space for redefining life, overcoming daily obstacles, developing new healthy life habits and engaging in active aging.
\end{abstract}

Descriptors: Occupational Therapy; Aged; Group Practice; Health education.

\section{RESUMEN}

Objetivo: Describir la experiencia de estudiantes de Terapia Ocupacional en la inserción de un grupo de convivencia de mayores de una comunidad localizada en la región central de Rio Grande do Sul, Brasil. Síntesis de los datos: La acción ha sido desarrollada en la Práctica Supervisada de Salud de la Comunidad en el período entre marzo y julio de 2015 realizado en el sexto período del curso de Terapia Ocupacional. Durante la interacción de los estudiantes con los participantes del grupo de convivencia se han realizado actividades para integrar las acciones de enseñanza, investigación y extensión universitaria dirigida para la mejoría de la calidad de vida y la condición de salud de las participantes a través de la prevención de agravios y complicaciones teniendo

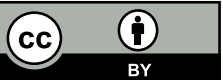


en cuenta las enfermedades crónicas no trasmisibles (ECNT) presentadas por la mayoría de los participantes del grupo de mayores. Conclusión: Las experiencias han evidenciado que los grupos de Terapia Ocupacional permitieron la identificación de las potencialidades y habilidades de una población poco valorada por su producción en la sociedad de manera que el grupo se ha constituido como un espacio para un nuevo sentido de las vidas, superación de dificultades del cotidiano, adquisición de hábitos de vida saludables y ayuda para el envejecimiento activo.

Descriptores: Terapia Ocupacional; Anciano; Práctica de Grupo; Educación en Salud.

\section{INTRODUÇÃO}

O Brasil manifesta mudanças em sua configuração populacional devido ao envelhecimento dos seus habitantes. O despreparo para essa alteração populacional aumenta a necessidade de um atendimento especializado, tanto no aspecto social quanto na saúde dos habitantes, a fim de oferecer aos idosos do país um envelhecimento digno e com melhor qualidade de vida(1).

Com a proximidade do envelhecimento para a população adulta, as doenças crônicas não transmissíveis (DCNT) também começam a se manifestar, podendo estar associado um desempenho insuficiente para a realização da maioria das atividades da vida diária (AVD), compreendidas como higiene pessoal e autocuidado, alimentação, banho e vestuário( ${ }^{(2)}$. O envelhecimento, quando associado à hipertensão arterial e outras doenças crônicas, é fator de risco elevado para o declínio nas funções cognitivas e riscos cardiovasculares, que afetam diretamente o cotidiano e a qualidade de vida do público idoso(3).

As doenças crônicas podem ser compreendidas por um conjunto de condições clínicas de causas múltiplas e com manifestações que ocorrem de forma gradual, de acordo com o indivíduo. Apresentam um prognóstico geralmente incerto e difícil de postergar, oscilando entre fases de agudização e de complicações graves, que podem gerar as incapacidades funcionais e físicas. Essas enfermidades trazem a necessidade de algumas mudanças nos hábitos de vida para que o indivíduo possa conviver com a doença, uma vez que sua cura nem sempre pode ser alcançada( ${ }^{(4)}$.

A Organização Mundial da Saúde (OMS) descreve em seu relatório de 2018 que são consideradas doenças crônicas não transmissíveis: as doenças cardiovasculares, as respiratórias crônicas, a diabetes mellitus (DM), o câncer, as doenças respiratórias e os transtornos mentais, sendo de duração continuada e motivada pelo uso excessivo do tabaco e álcool, inatividade física, má alimentação diária e entre outras causas ${ }^{(5)}$.

Dentre as patologias que mais comprometem e ocasionam representativas limitações no cotidiano dos indivíduos idosos, tem-se a hipertensão arterial sistêmica (HAS) e a DM em decorrência de suas complicações, como diminuição da capacidade visual, insuficiência cardíaca e demais comprometimentos físicos ${ }^{(2)}$.

A HAS e a DM são consideradas doenças crônicas muito prevalentes no Brasil, de forma que sua deteç̧ão precoce, controle e tratamento para a estabilização de seus níveis é indispensável para diminuir a gravidade em suas complicações. Além disso, a adoção de hábitos alimentares saudáveis, mudança no estilo de vida, prática de atividade física de forma regular e, na maioria das vezes, associação com tratamento medicamentoso, tem se mostrado muito eficaz nos estudos referentes a essas duas condições ${ }^{(6)}$.

A HAS é considerada uma síndrome associada a outras alterações metabólicas, como a obesidade, o aumento da resistência a insulina, a diabetes e as dislipidemias. A hipertensão também é considerada de alto risco para doenças coronarianas, doença vascular periférica, doença renal e insuficiência cardíaca, assim como também para as doenças cerebrovasculares, como o acidente vascular cerebral. São considerados hipertensos os sujeitos que manifestarem pressão arterial sistólica superior ou igual a $140 \mathrm{mmHg}$ e pressão arterial diastólica maior ou igual a $90 \mathrm{mmHg}$ durante duas ou mais situações ${ }^{(7)}$.

Por sua vez, a DM, no que lhe concerne, caracteriza-se por ser um distúrbio metabólico com hiperglicemia persistente resultante da ineficiência na secreção ou na resistência à insulina. Tanto a DM quando a HAS, quando associadas, somam maiores fatores de risco para as doenças cardiovasculares, um dos grandes problemas de saúde pública no Brasil, pois causa inúmeras hospitalizações devido a suas complicações e danos a órgãos alvos. Além desses danos, podem proporcionar uma perda significativa na qualidade de vida dos indivíduos devido à série de dificuldades que o indivíduo passa a $\operatorname{ter}^{(8)}$.

De acordo com a área de estudo e interesse, a qualidade de vida está associada a sinônimo de saúde, felicidade e satisfação pessoa, considerando-se a renda como conceito de satisfação para se atingir a uma qualidade de vida. No entanto, não há um consenso na literatura de uma única significação para o conceito de qualidade de vida, pois 
está ligado à percepção que o indivíduo adota sobre suas expectativas e objetivos, dentro dos valores segundo os quais vive ${ }^{(9)}$.

A DM e a HAS, por serem doenças crônicas, podem acompanhar os indivíduos pelo decorrer de suas vidas, no entanto seu impacto pode ser minimizado a partir da educação em saúde, buscando a conscientização sobre fatores de risco. Além disso, o tratamento adequado e a prática de atividade física podem contribuir para o idoso manter seu nível de independência e, consequentemente, evitar o sedentarismo, reduzindo gastos à saúde pública por complicações e tendo em seu benefício uma melhor qualidade de vida(10).

Os tratamentos indicados para a HAS e a DM geralmente estão unidos pelo tratamento medicamentoso e não medicamentoso. Cabe destacar que, conforme verificado em estudo, as medidas de tratamento não medicamentoso apresentam baixa frequência de adesão. Além disso, outras pesquisas ressaltam que somente medicamentos não são eficazes para o tratamento dessas doenças crônicas, sendo necessário associar as duas medidas, isto é, a atividade física e o controle da alimentação ${ }^{(11,12)}$, pois são medidas não farmacológicas que podem ajudar no controle dessas doenças, uma vez que a falta dessas duas medidas pode estar relacionada com $75 \%$ de novos casos de doenças crônicas ${ }^{(10)}$.

Considera-se atividade física todo movimento que seja realizado de forma voluntária pelo músculo esquelético causando um gasto metabólico de energia. Esse gasto de energia tem relação com altos níveis de bem-estar e satisfação, acarretando aumento nos padrões da capacidade funcional para desempenho de tarefas diárias ${ }^{(10)}$.

Além das medidas citadas acima, a falta de educação em saúde, principalmente em populações com nível de escolaridade menor que 12 anos, tem sido uma dificuldade para a compreensão da importância de hábitos saudáveis, tais como uma alimentação variada, uso do álcool e tabaco não nocivos, e a prática de atividade física. Através dessas constatações, têm se buscado chamar a atenção para a promoção de educação em saúde, principalmente entre o público idoso e pessoas de baixo nível de escolaridade ${ }^{(13)}$.

As formas não farmacológicas de controle da DM mais indicadas são: o controle do peso corporal, a prática de exercícios físicos regulares e a aquisição de hábitos saudáveis ${ }^{(14)}$.

A atenção especial para o controle da DM também se dá por meio da organização no consumo de alimentos. Os alimentos que possuem alto índice glicêmico devem ser substituídos pelo de baixo teor, e alimentos industrializados também devem ser evitados, pois contém diversos açúcares ocultos. É indicado pela Sociedade Brasileira de Diabetes a preferência por alimentos in natura, com o mínimo possível de processamento, e a ingestão de carboidratos advindos de vegetais, frutas, grãos integrais e legumes ${ }^{(8)}$.

Diversos profissionais, principalmente os da atenção básica de saúde, atuam por meio de trabalhos com grupos. A abordagem em grupo atua de forma terapêutica, pois possibilita um ambiente onde as emoções possam surgir, o contato comunicativo seja ampliado e também o resgate da ressocialização do idoso na constituição de novos vínculos e amizades ${ }^{(15)}$. Nessa perspectiva, muitas profissões utilizam o processo de avaliação, intervenção e análise dos resultados, contudo apenas a Terapia Ocupacional (TO) visa o uso das ocupações para a promoção da saúde, do bem-estar e participação de vida, facilitando a interação social entre os sujeitos com o ambiente e o contexto em que vivem e as ocupações que possuem ${ }^{(16)}$.

A Terapia Ocupacional trabalha com grupos no desempenho de atividades, nas quais os participantes envolvemse, simultaneamente, para realizar uma ou mais tarefas ou alguma atividade produtiva, criativa ou social, sendo essas atividades com um propósito terapêutico específico, estabelecido pelo terapeuta ocupacional(17).

Com base no enfoque da promoção da saúde e prevenção de doenças no campo da Terapia Ocupacional, acadêmicos do curso atuam em um projeto de extensão, a fim de desenvolver atividades de promoção e educação em saúde em um grupo de convivência voltado para mulheres com DCNT. Assim, este trabalho visa descrever a experiência de acadêmicas de Terapia Ocupacional na inserção de um grupo de convivência de idosos em uma comunidade localizada na região central do Rio Grande do Sul, Brasil.

\section{SÍNTESE DOS DADOS}

Trata-se de um relato de experiência oportunizado pelo Estágio Supervisionado em Saúde da Comunidade, ofertado aos acadêmicos do sexto semestre do curso de Terapia Ocupacional de uma universidade em Santa Maria, Rio Grande do Sul, Brasil.

A vivência ocorreu durante cinco meses, sendo realizada no período matutino, composto por três encontros semanais com o grupo, tendo duração de duas horas cada encontro. O local da realização do grupo era disponibilizado pela comunidade, localizado nas proximidades das residências das participantes. As ações desenvolvidas no local não geraram custos, portanto, as participantes não tiveram despesas durante a realização. 
O estágio estava vinculado a um projeto de extensão desenvolvido para mulheres de um bairro na região central do Rio Grande do Sul e das proximidades que tivessem interesse em inserir-se no grupo, sendo que a maioria das participantes era diabética e/ou hipertensa.

O projeto que propiciou essa vivência acadêmica foi composto por professores e acadêmicas de TO, desenvolvedores de ações, com o objetivo de propiciar maior atenção ao grupo utilizando-se dos recursos da dança e de atividades lúdicas e expressivas. Além disso, durante a experiência com as mulheres, a equipe desenvolveu grupos de estudos e debates sobre assuntos relacionados ao envelhecimento, saúde e qualidade de vida das pessoas, sendo realizadas dinâmicas de grupo, oficinas de arte e atividades de promoção à saúde de acordo com as demandas dos participantes do grupo.

\section{Contribuições do acadêmico ao grupo e atividades desenvolvidas}

A experiência dessa ação propiciou aos estudantes de Terapia Ocupacional o entendimento de sua contribuição enquanto profissional de saúde, na perspectiva grupal. As acadêmicas atuam no grupo como facilitadoras na realização das atividades e no processo de interação social entre os participantes do grupo de convivência, uma vez que auxiliam os participantes em suas dúvidas e dificuldades na realização das atividades.

As atividades desenvolvidas durante o período de vivência das acadêmicas consistiram em uma produção de salada de frutas e dinâmicas sobre sexualidade, relaxamento, estimulação cognitiva, dança, técnicas de expressividade, além de uma oficina de geração de renda.

A produção da salada de frutas, acima citada, teve enfoque para a utilização recomendada de diversificação de frutas e suas concentrações de açúcar, a fim de promover educação em saúde para o público diabético, buscando também informar sobre a necessidade de realizar uma alimentação saudável.

A atividade desenvolvida sobre sexualidade consistiu em uma roda de conversa com perguntas formuladas pelas acadêmicas sobre questões relacionadas ao tema. Essa oportunidade objetivou esclarecer mitos e dúvidas sobre o assunto. Durante essa vivência, surgiram algumas discussões e questionamentos sobre o uso de métodos contraceptivos e os cuidados necessários para evitar a contaminação e a transmissão das doenças sexualmente transmissíveis (DST) na faixa etária compreendida pelo público idoso.

As DST aumentaram significativamente no público idoso, devendo-se, na maioria das vezes, à falta de campanhas de prevenção que atinjam esse público, porque a sexualidade dos idosos está cercada por tabus e preconceitos da população ou, até mesmo, dos profissionais de saúde, por considerarem que não há uma vida sexual ativa nessa faixa etária(18).

A dinâmica associada à dança com balões procurou oferecer para as participantes um momento de interação, de cooperação mútua e de descontração. O recurso da dança instiga novas habilidades, com o uso de movimentos coordenados para conseguir segurar o balão e, ao mesmo tempo, dançar e escutar a música, contribuindo para o equilíbrio dinâmico e a coordenação motora ampla das participantes.

Outra proposta realizada com o grupo consistiu em uma técnica de relaxamento, visando à conscientização e percepção do próprio corpo, desenvolvida para a redução de estresse e autopercepção de cada paciente. A técnica de relaxamento referida foi embasada através do treinamento autógeno de Schultz, que busca alcançar um melhor nível de tranquilização, com redução de pensamentos depressivos, por meio de estímulos de visualização de imagens mentais do próprio corpo, que favorecem a admissão de um comportamento positivo e de maior rendimento corporal(19).

Além das atividades citadas, realizaram-se atividades de construção de objetos artesanais por meio de materiais reciclados. Essas ações propiciaram aos participantes aprendizados na construção de utensílios que poderiam ser utilizados como meio de geração de renda ou decoração de ambiente, como flores, garrafas, imãs e pufes.

Todas as atividades realizadas com o grupo foram previamente avaliadas por meio da análise da atividade, baseada nos estudos de autores importantes para a Terapia Ocupacional ${ }^{(20,21)}$. A análise de atividade busca identificar os objetivos, as potencialidades, a forma de execução, as habilidades necessárias e a importância de cada atividade. Essa análise também consiste na exposição da opinião de cada participante sobre as oficinas e seus significados ${ }^{(21)}$.

Dessa forma, entende-se por análise de atividade o processo de estudo e identificação dos elementos de cada atividade ${ }^{(21,22)}$. Essa avaliação possibilita ao terapeuta adaptar a atividade e utilizar-se dela como potencial terapêutico a partir da consideração de aspectos físicos, psicológicos, sociais, culturais e motivacionais dos sujeitos.

A Terapia Ocupacional, ao utilizar atividades como recursos terapêuticos, necessita dessa análise de atividade, a qual é baseada em um roteiro que auxiliará o terapeuta no direcionamento de sua ação, prevendo os aspectos biomecânicos e psicodinâmicos envolvidos com a atividade que será realizada( ${ }^{(20)}$.

A experiência ofertada às acadêmicas de Terapia Ocupacional corrobora o aprimoramento do conhecimento sobre análise de atividades, na compreensão dos cuidados necessários para atenção ao idoso e no desenvolvimento 
de grupos enquanto formação terapêutica ocupacional. Quando o terapeuta ocupacional desenvolve um grupo, ele assume a organização de um fazer, o qual é considerado terapêutico por possibilitar aos membros do grupo a vivência de novas formas de se relacionar e um fazer de forma autêntica, favorecendo a criação de um sentido para a ação que está sendo produzida enquanto grupo ${ }^{(23)}$.

\section{CONCLUSÃO}

Na construção do olhar do profissional de TO, as vivências necessitam ser desenvolvidas e aprimoradas para que os acadêmicos tenham maior qualidade e interação com seu futuro ambiente de atuação profissional.

Experiências, como a apresentada neste estudo, favorecem a maturidade, o raciocínio clínico e o comprometimento dos acadêmicos com outras pessoas no cuidado à saúde. A atuação prática dos acadêmicos durante a graduação, além de favorecer a interação profissional e a discussão de casos, também estimula a corresponsabilidade a ser assumida por cada profissional de saúde em educar e promover a saúde da população.

No que se refere à prática específica que este estudo apresenta, conclui-se que a intervenção da Terapia Ocupacional em grupo de pessoas idosas vai além da prevenção de doenças e agravos. As experiências evidenciaram que os grupos de Terapia Ocupacional permitiram a identificação de potencialidades e habilidades de uma população pouco valorizada produtivamente na sociedade, de forma que o grupo se constituiu como um espaço de ressignificação de vidas e superação de dificuldades cotidianas.

\section{CONFLITOS DE INTERESSE}

A descrição de experiência não possui fonte de financiamento ou auxílio institucional, e não possui relação que possa gerar conflitos de interesse.

\section{CONTRIBUIÇÕES}

Marciane Montagner Missio contribuiu com a escrita e revisão do artigo; Silvani Vargas Vieira contribuiu com a orientação do trabalho e a revisão do texto e referências.

\section{REFERÊNCIAS}

1. Miranda GMD, Mendes ACG, Silva ALA. O envelhecimento populacional brasileiro: desafios e consequências sociais atuais e futuras. Rev Bras Geriatr Gerontol. 2016;19(3):507-19.

2. Duarte GP, Uchôa-Figueiredo LR. A vida cotidiana e a qualidade de vida de pacientes atendidos na atenção primária de saúde. Cad Ter Ocup UFSCar. 2010;18(1):19-33.

3. Bezerra ALA, Bezerra DS, Pinto DS, Bonzi ARB, Pontes RMN, Veloso JAP. Perfil epidemiológico de idosos hipertensos no Brasil: uma revisão integrativa. Rev Med (São Paulo). 2018;97(1):103-7.

4. Brasil. Ministério da Saúde. Diretrizes para o cuidado das pessoas com doenças crônicas nas redes de atenção à saúde e nas linhas de cuidado prioritárias. Brasília: Ministério da Saúde; 2013.

5. Organização Mundial da Saúde. Saving lives, spending less: a strategic response to noncommunicable diseases.Geneva: WHO; 2018.

6. Stopa SR, Cesar CLG, Segri NJ, Alves MCGP, Barros MBA, Goldbaum M. Prevalência da hipertensão arterial, do diabetes mellitus e da adesão às medidas comportamentais no Município de São Paulo, Brasil, 2003-2015. Cad Saúde Pública. 2018;34(10):1-11.

7. Hernandez FH. Controle da hipertensão arterial com o tratamento não medicamentoso no posto de saúde da família. [Monografia]. Conselheiro Lafaiete (MG): Universidade Federal de Minas Gerais; 2016.

8. Sociedade Brasileira de Diabetes. Diretrizes da Sociedade Brasileira de Diabetes 2017-2018. São Paulo: Clannad; 2017.

9. Pereira EF, Teixeira CS, Santos A. Qualidade de vida: abordagens, conceitos e avaliação. Rev Bras Educ Fís Esp. 2012;26(2):241-50.

10. Agostini CM, Rodrigues VS, Guimarães AC, Damázio LCM, Vasconcelos NN. Análise do desempenho motor 
e do equilíbrio corporal de idosos ativos com hipertensão arterial e diabetes tipo 2. Rev Atenção Saúde. 2018;16(55):29-35.

11. Solbiati VP, Oliveira NRC, Teixeira CVS, Gomes RJ. Adesão ao tratamento para prevenir agravos relacionados à hipertensão arterial e ao diabetes. Rev Bras Obes Nutrição e Emagrec. 2018;12(73):629-33.

12. Brasil. Ministério da Saúde. Hipertensão arterial e diabetes mellitus: morbidade auto referida segundo VIGTEL, 2009. Cadastro de portadores do SIS-HIPERDIA, 2010. Brasilia: Ministério da Saúde; 2011.

13. Ribeiro KG, Andrade LOM, Aguiar JB, Moreira AEMM, Frota AC. Educação e saúde em uma região em situação de vulnerabilidade social: avanços e desafios para as políticas públicas. Interface Comun Saúde Educ. 2018;22(Supl 1):1387-98.

14. Brasil. Ministério da Saúde. Estratégia para o cuidado da pessoa com doença crônica: hipertensão arterial sistêmica. Brasília: Ministério da Saúde; 2013.

15. Soares DS, Braga JS, Alves SCA. Representação social de profissionais da área da saúde sobre grupos de convivência de idosos. Kaleidoscópio. 2012; 3:76-94.

16. Associação Americana de Terapia Ocupacional. Estrutura da prática da Terapia Ocupacional: domínio \& processo. 3a ed. Rev Ter Ocup Univ São Paulo. 2015;26(ed esp):1-49.

17. Hagedorn R. Ferramentas para prática em terapia ocupacional: uma abordagem estruturada aos conhecimentos e processos centrais. São Paulo: Roca; 2007.

18. Maschio MBM, Balbino AP, Souza PFR, Kalinke LP. Sexualidade na terceira idade: medidas de prevenção para doenças sexualmente transmissíveis e AIDS. Rev Gaúch Enferm. 2011;32(3):583-89.

19. Elias MTMNC. As Técnicas de relaxamento como instrumento terapêutico de enfermagem em pessoas com sofrimento mental [dissertação]. Lisboa: Escola Superior de Enfermagem de Lisboa; 2014.

20. Barbosa AA. Análise da atividade: adaptação de um roteiro para a implementação de tecnologia assistiva [trabalho de conclusão de curso]. Brasília: Universidade de Brasília; 2013.

21. Trombly CA. Terapia Ocupacional para a disfunção física. 2a ed. São Paulo: Santos; 1989.

22. Brown A. Groupwork. Londres: Heinemann; 1979.

23. Araújo AS, Kebbe LM. Estudo sobre grupos de terapia ocupacional para cuidadores de familiares de pacientes com esquizofrenia. Cad Ter Ocup UFSCar. 2014;22(1):97-108.

\section{Endereço para correspondência:}

Marciane Montagner Missio

Universidade Franciscana - UFN

Rua dos Andradas

Bairro: Centro

CEP: 97010-030 - Santa Maria - RS - Brasil

Email: marcianemissio@hotmail.com

Como citar: Missio MM, Vieira SP. Experiência em grupos de convivência de idosos: interfaces com a terapia ocupacional. Rev Bras Promoç Saúde. 2019;32:7436. 